\title{
Deformation of PDMS membrane and microcantilever by a water droplet: Comparison between Mooney-Rivlin and linear elastic constitutive models
}

\author{
Ying-Song Yu, Ya-Pu Zhao* \\ State Key Laboratory of Nonlinear Mechanics (LNM), Institute of Mechanics, Chinese Academy of Sciences, Beijing 100190, People's Republic of China
}

\section{A R T I C L E I N F O}

\section{Article history:}

Received 20 October 2008

Accepted 19 December 2008

Available online 25 December 2008

\section{Keywords:}

Mooney-Rivlin constitutive model

Hyperelastic material

PDMS

Membrane

Surface tension

Vertical component

Water droplet

Microcantilever

\begin{abstract}
A B S T R A C T
In this paper, we studied the role of vertical component of surface tension of a water droplet on the deformation of membranes and microcantilevers (MCLs) widely used in lab-on-a-chip and microand nano-electromechanical system (MEMS/NEMS). Firstly, a membrane made of a rubber-like material, poly(dimethylsiloxane) (PDMS), was considered. The deformation was investigated using the MooneyRivlin (MR) model and the linear elastic constitutive relation, respectively. By comparison between the numerical solutions with two different models, we found that the simple linear elastic model is accurate enough to describe such kind of problem, which would be quite convenient for engineering applications. Furthermore, based on small-deflection beam theory, the effect of a liquid droplet on the deflection of a MCL was also studied. The free-end deflection of the MCL was investigated by considering different cases like a cylindrical droplet, a spherical droplet centered on the MCL and a spherical droplet arbitrarily positioned on the MCL. Numerical simulations demonstrated that the deflection might not be neglected, and showed good agreement with our theoretical analyses.
\end{abstract}

(c) 2008 Elsevier Inc. All rights reserved.

\section{Introduction}

Since poly(dimethylsiloxane) (PDMS) has many advantages, e.g., (1) good biocompatibility; (2) nontoxic and optically transparent; (3) easily fabricated, it has been widely used in lab-on-a-chip [1-6] and some microfluidic devices [7-13], etc.

Up to now, researchers have made many experiments about the mechanical properties of PDMS. Lötters et al. [14] first used PDMS PS851 from ABCR to measure the shear modulus and further studied relationship versus frequency and temperature. Armani et al. [15] utilized PDMS from Dow Corning to measure the Young's modulus with different mixing volume ratios of the curing agent and the polymer. Huang and Anand [16] carried out experiments on traditional macroscale specimens and thin-film specimens of PDMS with three varying ratios of monomer and curing agent to study the nonlinear mechanical behavior of PDMS material. Recently, Schneider and co-workers [17] have studied the mechanical properties of PDMS (weight ratio: 10:1) with different thinner concentrations (the thinner was added in order to get a lower viscosity) and the elastic modulus for Sylgard 184 without adding thinner against temperature and strain rate, respectively.

As a matter of fact, PDMS is a kind of rubber-like material with nearly or purely incompressible property. As a common knowledge,

\footnotetext{
* Corresponding author. Fax: +86 1062561284

E-mail address: yzhao@imech.ac.cn (Y.-P. Zhao).
}

one of the most widely used models for such a material is the Mooney-Rivlin (MR) constitutive model. Mooney [18] and Rivlin and Saunders [19] developed the first hyperelastic models. Many other hyperelastic models have since been developed. Hyperelastic models can be classified as [20]:

(1) Phenomenological descriptions of observed behavior

- Polynomial model: Subject to the regularity assumption that strain energy function $W$ is continuously differentiable several times with respect to three strain invariants $I_{1}, I_{2}$ and $I_{3}$, the strain energy function is assumed to be an polynomial form of $\left(I_{1}-3\right),\left(I_{2}-3\right)$ and $\left(I_{3}-1\right)$. For an incompressible material $I_{3}=1$ and $W$ depends on only two independent deformation invariants [21].

- MR model: The strain energy function of this model is the first-order polynomial form; more exactly, it is only linear functions of the invariants $I_{1}$ and $I_{2}$. It is the most widely used hyperelastic constitutive relation due to its simplicity and convenience for practical use.

- Ogden model: Ogden in 1972 deduced a hyperelastic constitutive model for large deformations of incompressible rubber-like solids. The strain energy is expressed as a function of the principal stretches. For particular values of material constants, the Ogden model will reduce to either the Neo-Hookean solid or the MR material.

- Yeoh model: The Yeoh (1993) model depends only on the first strain invariant $I_{1}$, it applies to the characterization 
of elastic properties of carbon-black filled rubber vulcanizates. The Yeoh model is also called the reduced polynomial model.

(2) Mechanistic models deriving from arguments about underlying structure of the material

- Arruda-Boyce model: The constitutive model for the large stretch behavior of rubber elastic materials is presented by Arruda and Boyce (1993). Also sometimes called the eightchain model because it was derived by idealizing a polymer as eight elastic chains inside a volume element. The strain stress function is based on an eight chain representation of the macromolecular structure of the rubber.

- Neo-Hookean model: The model was derived from the statistical mechanics of a molecular chain network characteristic of the amorphous structure of rubber-like material by Treloar in 1943. It is the simplest model of rubber-like elastic response, in which only the term of $I_{1}$ is considered. Thus, Neo-Hookean material is a particular kind of MR material.

(3) Hybrids of phenomenological and mechanistic models

- Gent model: The strain energy density in the Gent model (1996) is a simple logarithmic function of the first strain invariant $I_{1}$, and involves two material constants.

For the relevant applications, Feng and Huang [22] and Pamplona et al. [23] used the MR model to investigate the large deformations of cylindrical membrane under internal pressure with the software ABAQUS and made comparison between the experimental and numerical results for the membrane under traction to identify the material parameters of the model. Three-dimensional (3D) solid element analysis and the membrane approximated analysis employing the hyperelastic model were developed for the simulation of the thermoforming process by Nam et al. [24]. Bellamy et al. [25] have recently simulated the mechanical behavior of the polymer up to $50 \%$ strain using MR model and found that the solutions were in excellent agreement with analytical and experimental results. Dong et al. [26] used the MR model to obtain the critical material parameters of polymethylmethacrylate (PMMA) with experimental verification, and then derived the major material parametric functions at temperatures between $150^{\circ} \mathrm{C}$ and $190^{\circ} \mathrm{C}$.

When there is a water droplet on the surface of PDMS membrane with a rigid substrate, the PDMS material was simply regarded as a linear elastic material because the deformation is very small, and then numerical simulation was carried out for the elastic deformation of the membrane due to the vertical components of liquid-vapor surface tension [27]. However, linear elastic material model is only a first-order approximation for a hyperelastic material like PDMS. The main objective of the present paper is to make a guideline for the accuracy range for the linear elastic model to simulate the elastic deformation of PDMS under the action of a water droplet.

Determination of the materials constants in the MR model is a prerequisite to use this model for the numerical simulation. Fortunately, there are some literatures on for relevant experiments [28-30]. In addition, Gent [31] has proposed some other methods to get the material constants.

Besides, we continued to study the effect of a liquid droplet on the deformation of a MCL. For macro structures, the deformation induced by liquid droplets may be neglected simply since the surface tension and the Laplace pressure are negligible compared to the rigidity of the solid body; while in MEMS/NEMS, vertical displacement induced by them may be several to hundreds nanometers and even hundreds micrometers when the materials are very soft. In such cases, the deformation cannot be neglected anymore. For example, if there are several microdrops on the MCL of an atomic force microscopy (AFM), they may decrease the precision of AFM. Therefore, it appears significant to study the effect of liquid droplets on MCL.

During the recent years, some researchers have made some relevant investigations on the deflection of MCL due to microdrops, as shown in Fig. 9. Jensenius et al. integrated resistors on flexible cantilevers to monitor the cantilever deflection [32]. Bonaccurso et al. studied microdrops on AFM cantilevers theoretically and experimentally $[33,34]$. Some researchers studied the influence of nanobubbles on the bending of MCLs [35,36]. Recently, Bonaccurso et al. developed a FEM model for the bending of a cantilever and measured the bending versus time [37]. Zheng et al. used the energy method to study the directional movement of liquid droplets on a microbeam with a varying or gradient stiffness and found that the droplet will move to the softer end of the beam [38].

In the first part of this paper, we first introduced the procedure to determine the material constants in the MR model. Then we used the least square method (LSM) to obtain the material constants from experimental data in Ref. [16]. At last, both the linear elastic and the MR constitutive models were used to numerically simulate the deformation of PDMS membrane induced by a water droplet. And we found the solutions with a linear elastic model are in excellent agreement with that by using the MR model for such problem. In the latter part, we theoretically analyzed the effect of a liquid droplet on the deflection of a MCL and gave some numerical simulations to demonstrate the deflection might not be neglected.

\section{MR model and determination of material constants}

A hyperelastic material is an ideally elastic material for which the stress-strain relationship derives from a strain energy density function. The strain energy density function, $W$, for PDMS is the function of the three strain invariants $I_{1}, I_{2}$ and $I_{3}$, i.e. [20],

$W=W\left(I_{1}, I_{2}, I_{3}\right)$,

where $I_{1}=\operatorname{tr} \mathbf{C}, I_{2}=\left[(\operatorname{tr} \mathbf{C})^{2}-\operatorname{tr} \mathbf{C}^{2}\right] / 2$.

Then, corresponding stress can be expressed as

$\mathbf{S}=\frac{\partial W}{\partial \mathbf{E}}=2 \frac{\partial W}{\partial \mathbf{C}}$,

where $\mathbf{S}$ is the second Piola-Kirchhoff stress tensor, $\mathbf{E}$ is the Green strain tensor and $\mathbf{C}$ is the right Cauchy-Green deformation tensor. $\mathbf{E}$ and $\mathbf{C}$ satisfy the following relationship:

$\mathbf{E}=\frac{1}{2}(\mathbf{C}-\mathbf{I})$,

where $\mathbf{I}$ is the second-order unit tensor.

To ensure incompressibility of a hyperelastic material, the constraint $I_{3}=1$ must be fulfilled. The strain-energy function, $W$, can be written as a polynomial function of $\left(I_{1}-3\right)$ and $\left(I_{2}-3\right)[18,21]$

$W=\sum_{i=0}^{\infty} \sum_{j=0}^{\infty} c_{i j}\left(I_{1}-3\right)^{i}\left(I_{2}-3\right)^{j}$,

where $c_{i j}$ are empirically determined material parameters.

Because the deformation of PDMS material in the present paper is very small under the action of a water droplet, two-parameter MR model is used as follows [18]:

$W=c_{1}\left(I_{1}-3\right)+c_{2}\left(I_{2}-3\right)$,

where $c_{1}$ and $c_{2}$ are material constants. The model has an applicable strain of about $100 \%$ in tension and $30 \%$ in compression. Then, the constitutive equation for hyperelastic incompressible materials can be expressed as: 
$\boldsymbol{\sigma}=-p \mathbf{I}+2\left(c_{1} \mathbf{C}-c_{2} \mathbf{C}^{-1}\right)$,

where $\sigma$ is the Cauchy stress tensor, and $p$ is the hydrostatic pressure.

If the principal directions of deformation are supposed as the directions of coordinate, then Cauchy strain tensor can be expressed as:

$\mathbf{C}=\left[\begin{array}{ccc}\lambda_{1}^{2} & 0 & 0 \\ 0 & \lambda_{2}^{2} & 0 \\ 0 & 0 & \lambda_{3}^{2}\end{array}\right]$,

where $\lambda_{i}$ is the principal stretch ratios in $i$ th direction, $\lambda_{i}=1+\varepsilon_{i}$, $\varepsilon_{i}$ is the principal value of engineer strain in $i$ th direction, so the invariants of $C_{i j}$ can be expressed as:

$\left\{\begin{array}{l}I_{1}=\lambda_{1}^{2}+\lambda_{2}^{2}+\lambda_{3}^{2}, \\ I_{2}=\lambda_{1}^{2} \lambda_{2}^{2}+\lambda_{2}^{2} \lambda_{3}^{2}+\lambda_{3}^{2} \lambda_{1}^{2}, \\ I_{3}=\lambda_{1}^{2} \lambda_{2}^{2} \lambda_{3}^{2} .\end{array}\right.$

For uniaxial tension (i.e., $\lambda_{1}=\lambda, \lambda_{2}=\lambda_{3}=\lambda^{-1 / 2}$ ), $\sigma_{11}=\sigma$, $\sigma_{22}=\sigma_{33}=0$, then the constitutive relation (6) becomes

$\sigma=2\left(\lambda^{2}-\lambda^{-1}\right)\left(c_{1}+c_{2} \lambda^{-1}\right)$. [26]:

When $\lambda \rightarrow 1$, we can get the initial Young's modulus as follows

$\lim _{\lambda \rightarrow 1} \frac{\partial \sigma_{11}}{\partial \lambda}=6\left(c_{1}+c_{2}\right)$,

where $E$ is Young's modulus for infinitesimal deformation, and the initial shear modulus is

$G=\frac{E}{2(1+v)}=2\left(c_{1}+c_{2}\right)$,

where $v$ is the Poisson's ratio, and $v$ is 0.5 for PDMS.

From Eq. (9), we know that the plot of $\frac{\sigma}{2\left(\lambda^{2}-\lambda^{-1}\right)}$ versus $\lambda^{-1}$ is a linear line, then we can easily get $c_{1}$ and $c_{2}$ from the intercept and slope [28-30], respectively. However, the plot is not exactly a linear line. Therefore, we introduce another method-LSM to obtain these parameters.

If uniaxial tension experimental data were available, then we can draw the plot of engineering stress $S_{i}$ (subscript $i$ denotes the sequence number of data points) versus stretch ratio $\lambda_{i}$, and get the corresponding theoretical Cauchy stress $\tilde{\sigma}_{i}\left(c_{j}\right)(j=1,2)$, from Eq. (9). And the real stresses obtained from experiments are $\sigma_{i}=$ $\lambda_{i} S_{i}$, then we use the LSM to determine the material constants $c_{j}$. The sum of the squared error is defined by:

$R=\sum_{i=1}^{n}\left[\sigma_{i}-\tilde{\sigma}_{i}\left(c_{j}\right)\right]^{2}$.

As we know, to minimize the squared error $R$, we just only let its variation be zero: $\delta R=0$. This produces two simultaneous equations:

$\frac{\partial R}{\partial c_{1}}=0, \quad \frac{\partial R}{\partial c_{2}}=0$.

Then, the material constants can be solved from Eq. (13).

Huang and Anand [16] conducted large-deformation uniaxial tension experiments on traditional macroscale specimens of PDMS with varying ratios of monomer: curing agent (5:1, 20:1), and the experimentally determined macroscale tension engineering stress versus stretch curves are shown in Fig. 1.

Using LSM, we can get: $c_{1}=0.7953 \mathrm{MPa}, c_{2}=-0.6318 \mathrm{MPa}$, and the initial Young's modulus is thus $E=0.9810 \mathrm{MPa}$ when varying ratio is $5: 1$; and $c_{1}=0.07406 \mathrm{MPa}, c_{2}=0.008340 \mathrm{MPa}$, and $E=0.4944 \mathrm{MPa}$ for $20: 1$.

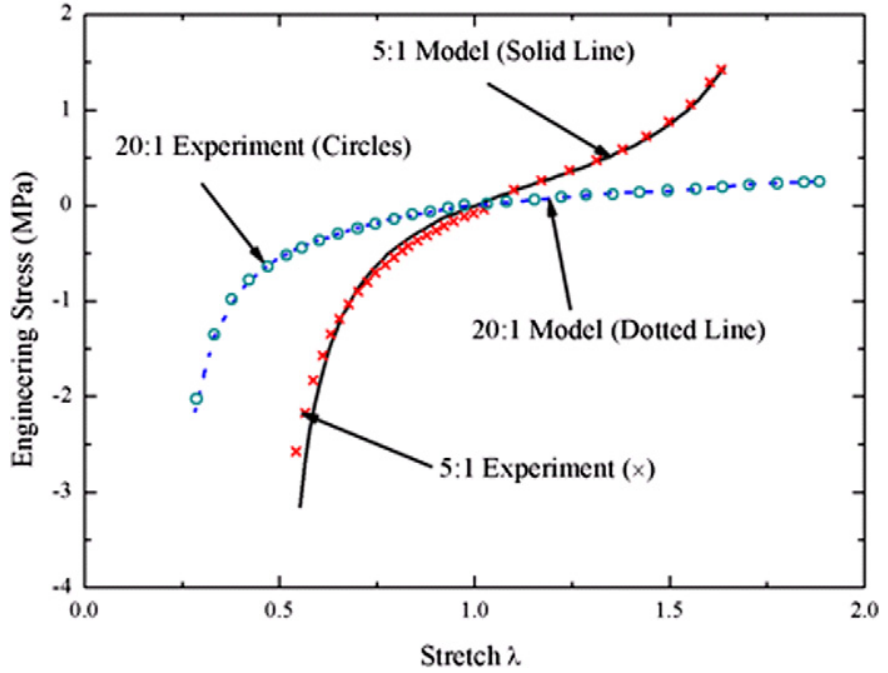

Fig. 1. Engineering stress versus stretch curves for 5:1 and 20:1 PDMS [16].

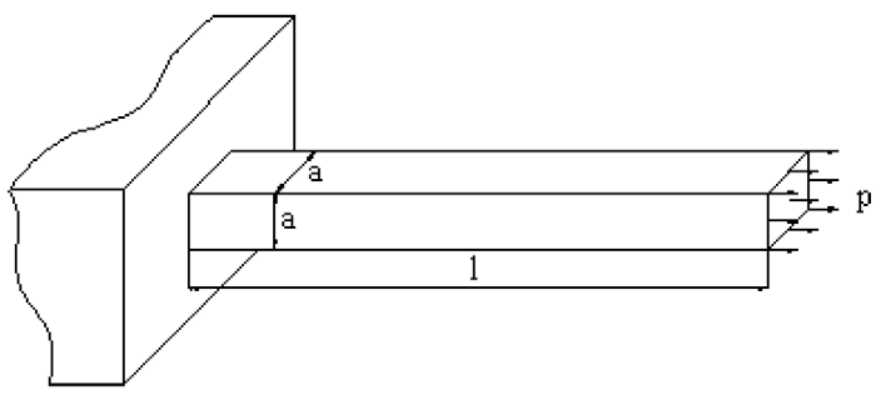

Fig. 2. Sketch of a PDMS beam loaded by a unit tension.

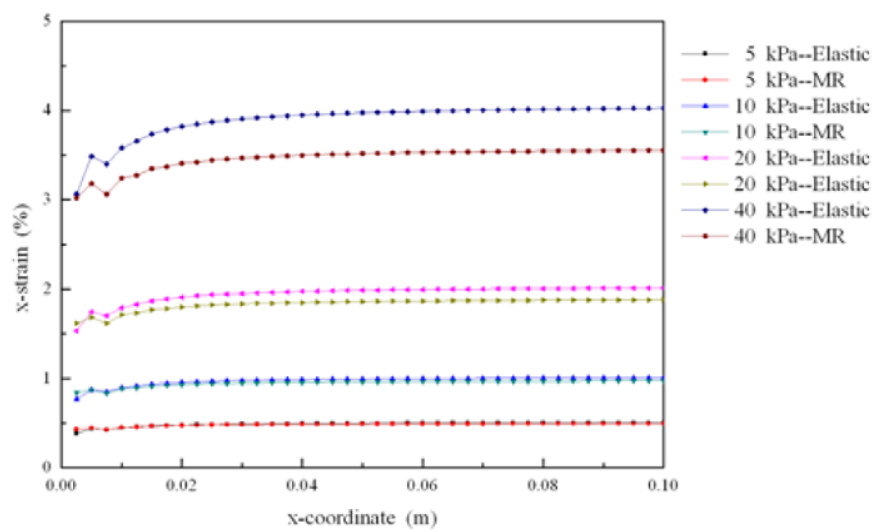

Fig. 3. Strains in $x$-direction with different loads and models.

\section{Numerical simulation and comparison for PDMS membrane}

Because the surface tension and Laplace pressure are very little compared to the material rigidity, the deformation of PDMS membrane may be relatively small. In order to make sure the accuracy range of the linear elastic model for the first-order approximation of the MR model, we first consider a PDMS beam (varying ratio: 5:1) with dimensions $0.1 \mathrm{~m} \times 0.01 \mathrm{~m} \times 0.01 \mathrm{~m}$ loaded by a unit tension $p$ at the end $x=0.1 \mathrm{~m}$, and clamped at the end $x=0$, as shown in Fig. 2. For the ratio of 5:1, using both linear elastic model and MR model, we can get the strains in $x$-direction as shown in Fig. 3 under the actions $p=5 \mathrm{kPa}, 10 \mathrm{kPa}, 20 \mathrm{kPa}$ and $40 \mathrm{kPa}$, respectively. 


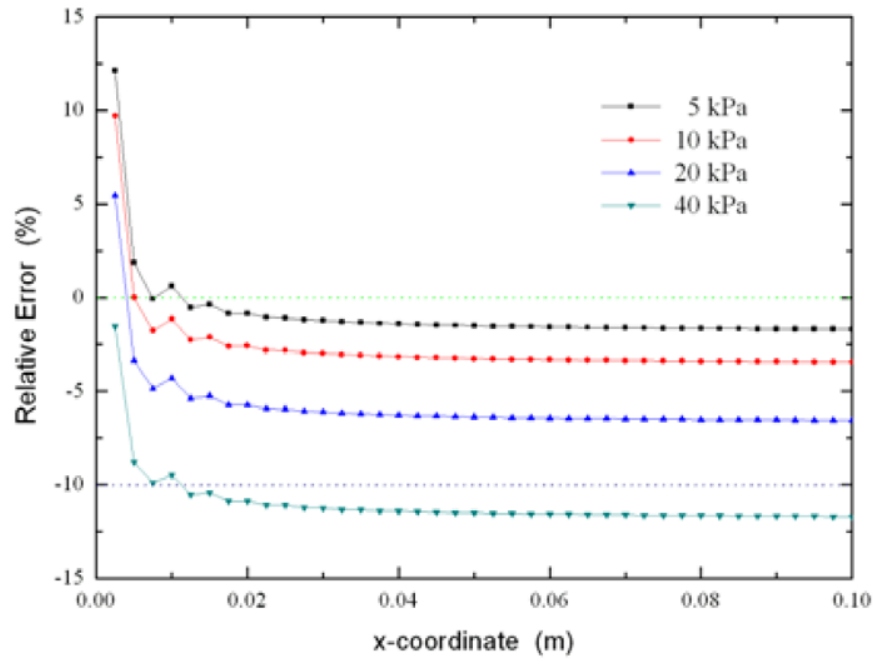

Fig. 4. Relative errors of strains in $x$-direction under different loads.

The relative error of strains calculated in different models can be defined as:

$e=\frac{\varepsilon_{\mathrm{MR}}-\varepsilon_{\text {Elastic }}}{\varepsilon_{\text {Elastic }}} \times 100 \%$.

And we make a plot of the relative error versus $x$-coordinate as shown in Fig. 4. From Fig. 4, we can easily find that the relative errors increase with increasing applied load, and when the maximum strain with MR model is approximately $1.8 \%$, the relative error is about $5 \%$, that is to say, in such circumstance, PDMS can be regarded as a linear elastic material. However, when the maximum strain with MR model is about 3.5\%, the maximum strain with linear elastic model is a little more than $4 \%$ and the relative error is larger than $10 \%$, then the MR model should be adopted. Then, we conclude that when the relative error is no more than $10 \%$, linear elastic model will be valid for some engineering applications; otherwise, the MR model must be used.

Now we consider the role of vertical component of surface tension of water droplet on the deformation of PDMS membrane by using both linear elastic and MR models. First, PDMS membranes (polydimethylsiloxane, Sylgard 184, Dow Corning, USA; ratio of the base to curing agent $=5: 1,20: 1$ ) which we used were vacuumed for an hour to remove the trapped air-bubbles, and then were cured about 20 hours at $75^{\circ} \mathrm{C}$ in a class- 1000 clean room. Then, the contact angles were measured with the OCA20 system (with precision $\pm 0.1^{\circ}$ ) from Dataphysics, Germany. A $3 \mu \mathrm{L}$ water droplet was placed on the surface of PDMS membrane, in order to get a reasonable contact angle, we repeated it at different places for six times, measured the contact angles, and then got the averaged contact angles $\theta=109.4^{\circ}$ for the ratio $5: 1$ and $\theta=118.0^{\circ}$ for $20: 1$ as shown in Fig. 5, respectively. The surface tension of water at $25^{\circ} \mathrm{C}$ is $\gamma_{l v}=0.072 \mathrm{~N} / \mathrm{m}$. Supposed the water droplet is spherical and the contact region is a circle with radius $a$, then the volume of droplet can be expressed as:

$V=\frac{1}{3} \pi a^{3} \frac{2-\cos \theta\left(3-\cos ^{2} \theta\right)}{\sin ^{3} \theta}$.

Then we can get $a=0.93 \mathrm{~mm}$ for $5: 1$ and $a=0.84 \mathrm{~mm}$ for $20: 1$, respectively. Then we know that the weight of the droplet can be neglected since the diameter of the water droplet is much less than the capillary length of water, $2.7 \mathrm{~mm}$ [27].

Suppose the PDMS membrane is $10.0 \mathrm{~mm}$ in diameter and $0.5 \mathrm{~mm}, 1.0 \mathrm{~mm}$ and $2.0 \mathrm{~mm}$ thick. As the case in Ref. [27], the membrane will deform due to surface tension and Laplace pressure, as shown in Fig. 6. Using the commercial code ANSYS, both

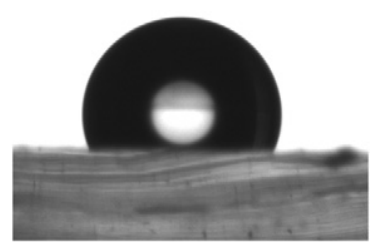

(a)

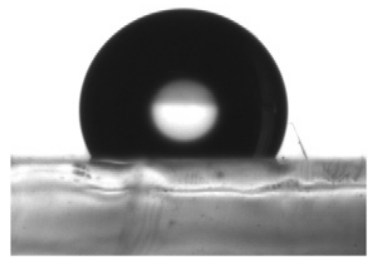

(b)

Fig. 5. Contact angle of water droplet on the surface of PDMS membrane: (a) 5:1; (b) $20: 1$.

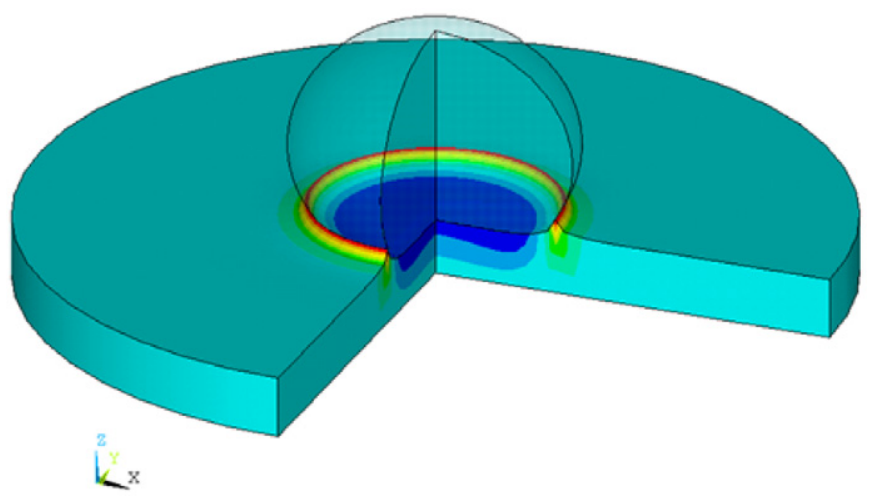

Fig. 6. Sketch of deformation of PDMS membrane induced by a water droplet.

the linear elastic (in this model, Poisson's ratio of PDMS is 0.5 and 0.499 was used in the numerical simulations) and the MR models were used to simulate the deformation. The vertical displacements on the surface of PDMS membrane are shown in Fig. 7.

From Fig. 7, we found that no matter which model was used, the maximum vertical displacement decreases a little while the thickness changed from $0.5 \mathrm{~mm}$ to $2.0 \mathrm{~mm}$. In addition, since there is no external force in $x$-direction and $y$-direction, the strains in those directions are rather small. That is, the maximum strain decreases with increasing thickness of PDMS membrane. So we compared vertical displacements calculated with these models when the thickness is $0.5 \mathrm{~mm}$, as shown in Fig. 8, from which, we can easily find that the solutions with different models fit well except the place nearest where the surface tension is applied, that is to say, we can use the linear elastic model rather than more complicated MR model to solve such a question for enough accuracy, for the maximum strain is $\varepsilon_{\max }<0.15 \mu \mathrm{m} / 0.5 \mathrm{~mm}=3.0 \times 10^{-4} \ll$ $1.5 \%$ for the varying ratio $5: 1$ and $\varepsilon_{\max }<0.30 \mu \mathrm{m} / 0.5 \mathrm{~mm}=$ $6.0 \times 10^{-4} \ll 1.5 \%$ for the varying ratio $20: 1$. When the thickness of PDMS membrane increases, the maximum of vertical displacement decreases reversely, then the maximum strain will be smaller, therefore, the linear elastic model is still valid.

\section{Deflection of MCLs induced by liquid droplets}

In this section, we studied the effect of one or several water droplets sitting on a MCL on the deflection of the MCL due to the 


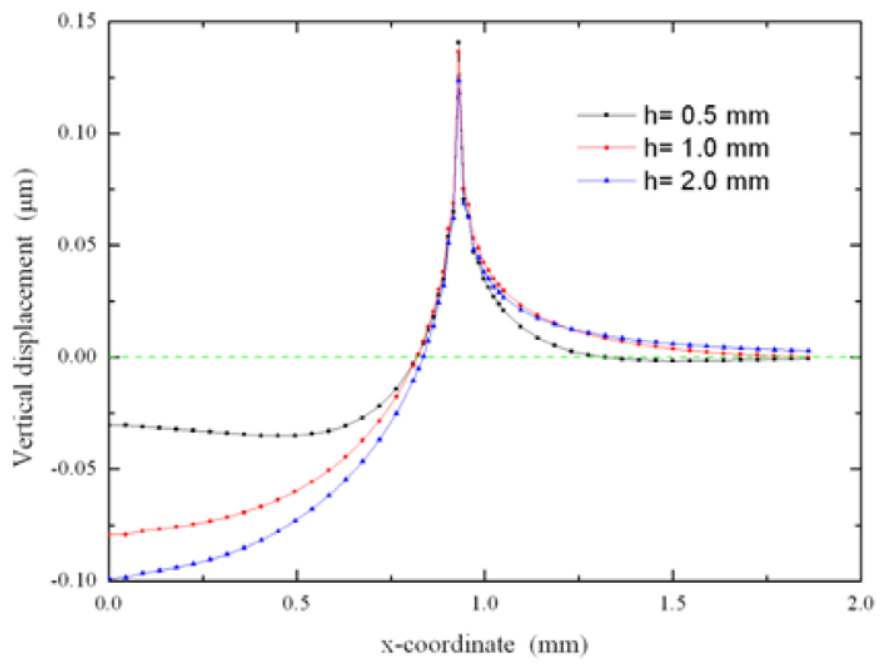

(a)

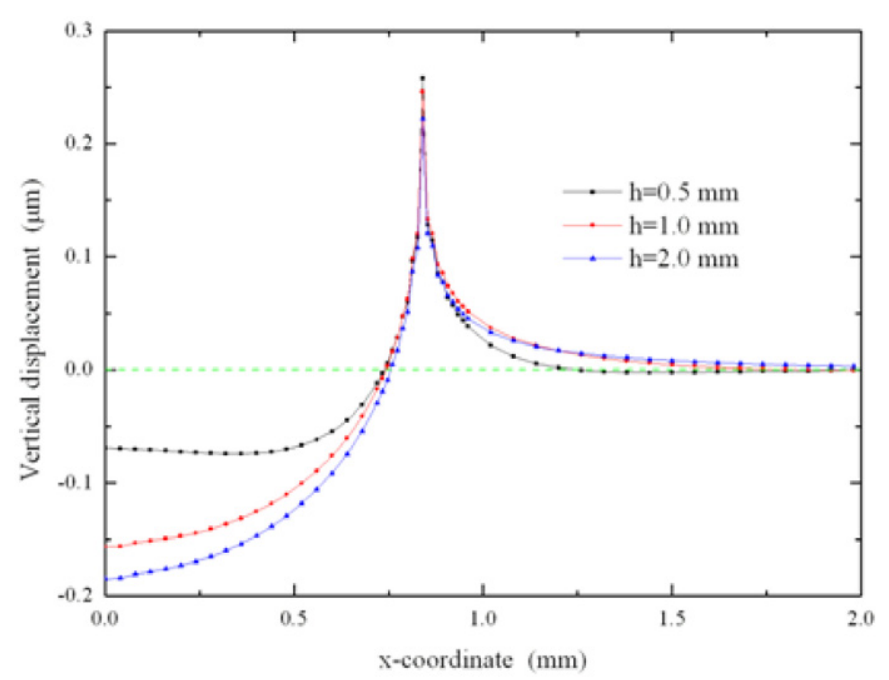

(c)

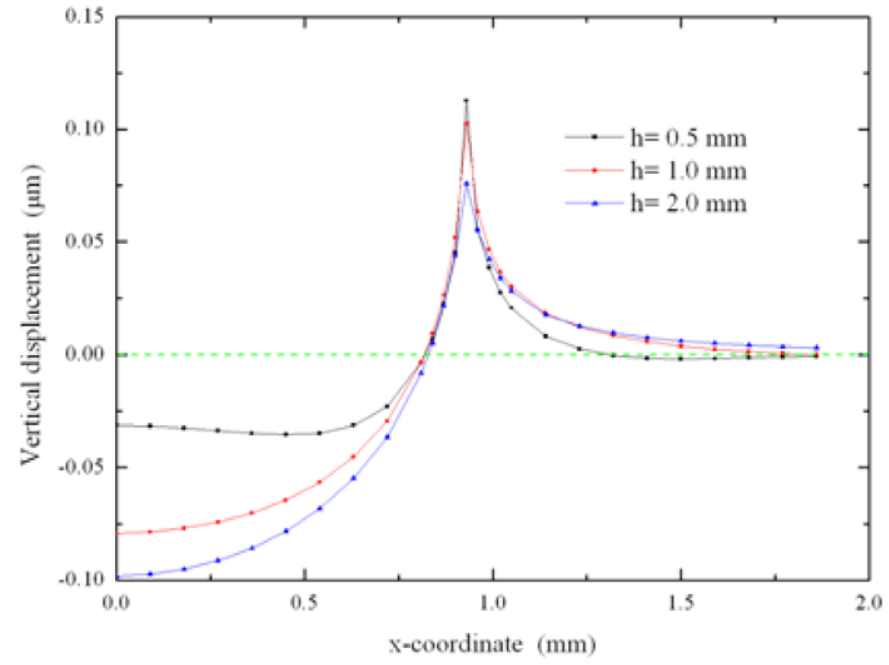

(b)

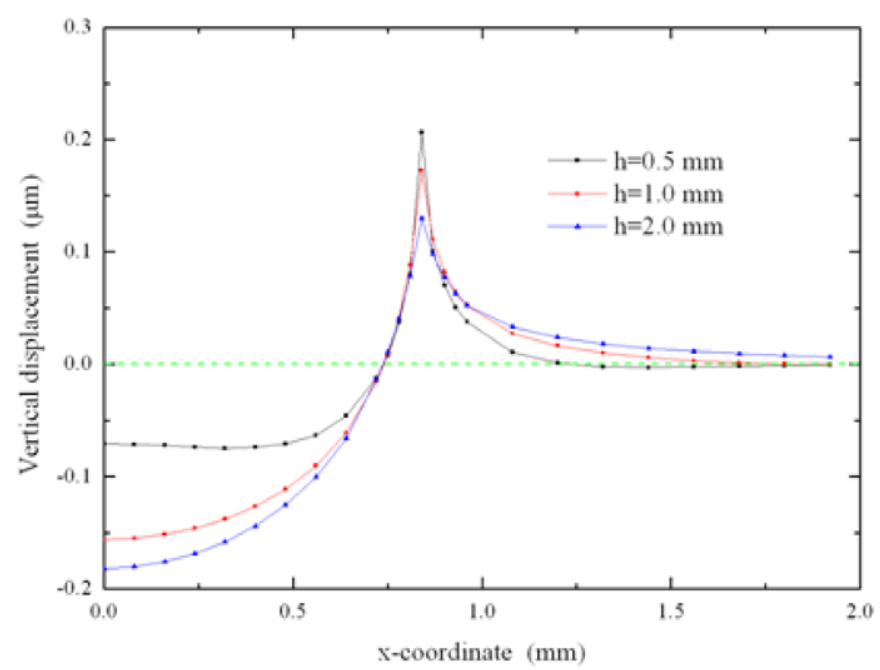

(d)

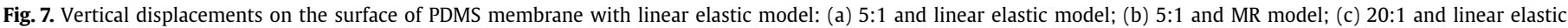
model; and (d) 20:1 and MR model.

vertical component of the surface tension and the Laplace pressure in some cases, basing on small-deflection beam theory.

\subsection{Cylindrical droplet on $M C L$}

Now we considered one or several water droplets placed on a MCL as shown in Fig. 10. First, we suppose the droplet is ideally cylindrical [37] and the contact angle is $\theta$. The wetted area is $\mathrm{A}=2 w a=2 w r \sin \theta$, where $r$ is the curvature radius of the water droplet. On the wetted area acts a Laplace pressure $p=\gamma_{l v} / r$, which is compensated by the vertical component of the liquidvapor interface tension $\gamma_{l v} \sin \theta$ acting on the contact line. The torque induced by the droplets at a given position is expressed as

$M(x)= \begin{cases}0, & x \leqslant-a, \\ \gamma_{l v} w \sin \theta \frac{a^{2}-x^{2}}{2 a}, & -a \leqslant x \leqslant a, \\ 0, & a \leqslant x .\end{cases}$

The relationship between the torque and the deflection of the MCL is

$M(x)=E I \frac{d^{2} z}{d x^{2}}$, where $I=w d^{3} / 12$. Since the external torsion is 0 when $x \leqslant a$, the inclination and deflection at the field are both equal to 0 . Integrating Eq. (17) once and using the boundary condition $(d z / d x)(x=$ $-a)=0$, we can get the rotary angle of the MCL as follows:

$\frac{d z}{d x}=\frac{\gamma_{l v} w \sin \theta}{6 E I a}\left(-x^{3}+3 a^{2} x+2 a^{3}\right), \quad-a \leqslant x \leqslant a$.

Integrating Eq. (18) once and using the boundary condition $z(x=$ $-a)=0$, we obtain

$z=\frac{\gamma_{l v} w \sin \theta}{24 E I a}\left(-x^{4}+6 a^{2} x^{2}+8 a^{2} x+3 a^{4}\right), \quad-a \leqslant x \leqslant a$.

Then we know at $x=a$, the inclination and deflection are, respectively,

$\frac{d z}{d x}(x=a)=\frac{8 \gamma_{l v} a^{2} \sin \theta}{E d^{3}}$,

and

$z(x=a)=\frac{8 \gamma_{l v} a^{3} \sin \theta}{E d^{3}}$.

For $x \geqslant a$, since the external forces and bending moments are all zero, then the inclination is constant and equal to that at $x=a$, therefore, the deflection at the free end is 


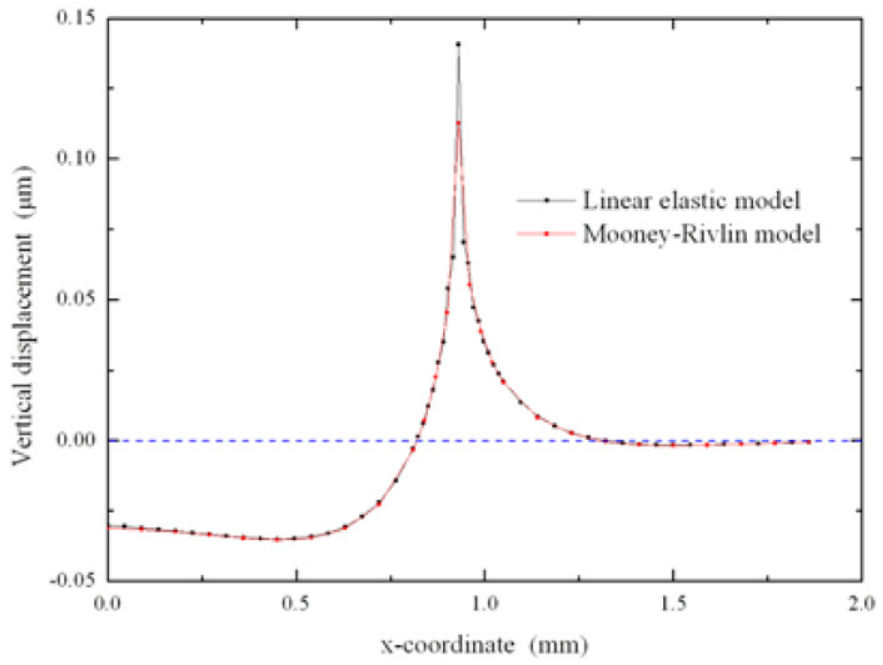

(a)

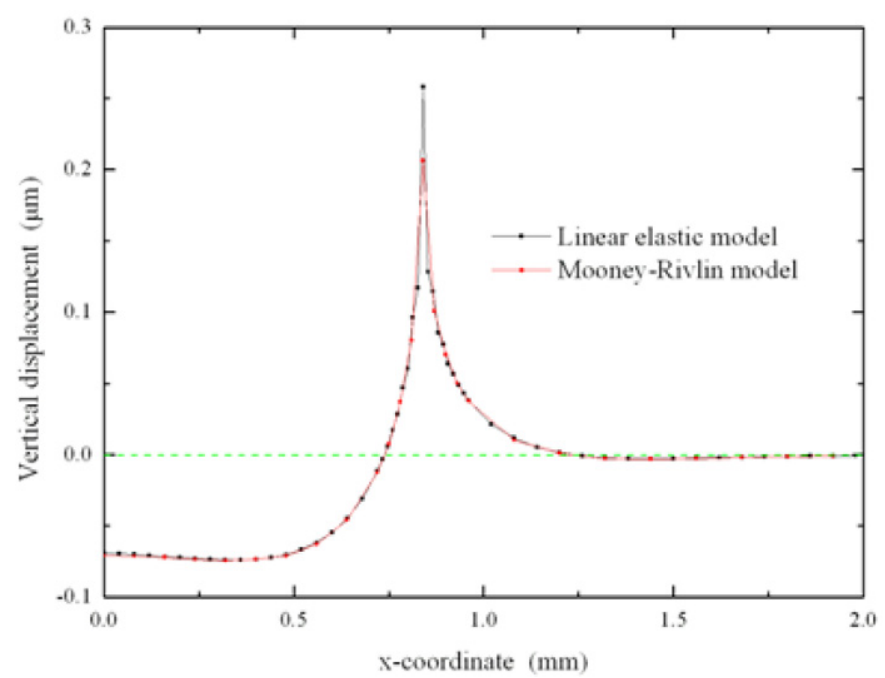

(b)

Fig. 8. Vertical displacements with different models: (a) $5: 1$ and $h=0.5 \mathrm{~mm}$; (b) $20: 1$ and $h=0.5 \mathrm{~mm}$.

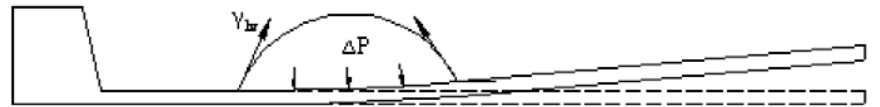

Fig. 9. Sketch of the deformation of a MCL induced by a liquid droplet.

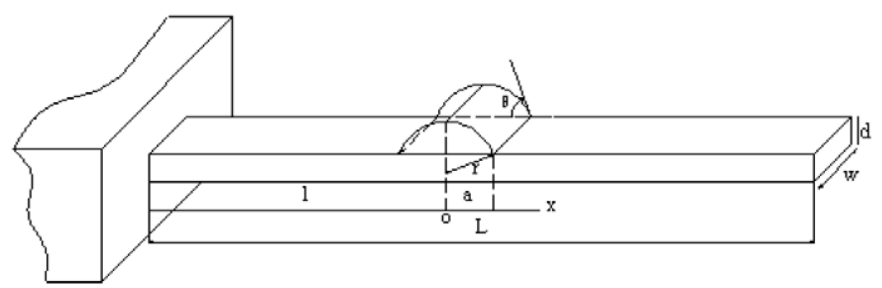

Fig. 10. Cylindrical water droplet placed on a MCL.

$z(x=L-l)=\frac{8 \gamma_{l v} a^{2} \sin \theta}{E d^{3}}(L-l)$.

Then we can easily see that the inclination and deflection at $x \geqslant a$ have nothing with the width $w$, and both of them are proportional to $\gamma_{l v} a^{2} \sin \theta /\left(E d^{3}\right)$. Moreover, the free-end deflection will increase when the droplet approaches to the fixed end.

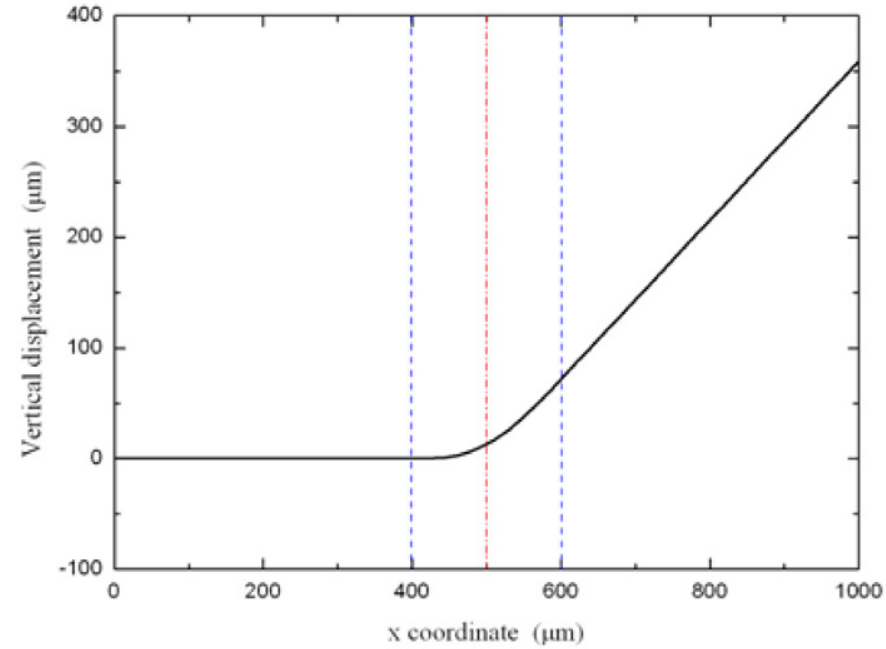

Fig. 11. Vertical displacement of a MCL induced by a cylindrical droplet.

The elastic deformation of a MCL induced by a water droplet was simulated by using FEM. The parameters used are listed as follows:

$\gamma_{l v}=0.072 \mathrm{~N} / \mathrm{m}, \quad E=1 \mathrm{MPa}, \quad \theta=90^{\circ}$,

$a=r=100 \mu \mathrm{m}, \quad a=100 \mu \mathrm{m}, \quad l=500 \mu \mathrm{m}$,

$L=1000 \mu \mathrm{m}, \quad d=20 \mu \mathrm{m}, \quad w=50 \mu \mathrm{m}$.

According to the above analysis, we know that the deflection at the free end is

$z(x=L-l)=\frac{8 \gamma_{l v} a^{2} \sin \theta}{E d^{3}}(L-l)=360 \mu \mathrm{m}$,

which is quite large for the MCL. The numerical results of the deformation of the MCL are shown in Fig. 11, the deflection at the free end is calculated as $358.02 \mu \mathrm{m}$.

\subsection{Spherical droplet sitting at the midline of $M C L$}

Now we first consider a spherical droplet and the center of the contact area is just located at the midline of the upper surface of the MCL. For this case, since the external forces are symmetrical to the midline, there are only torques which make the cantilever bending, the torques are given as follows:

$$
\begin{aligned}
M_{1} & =2 \int_{0}^{\varphi} \gamma_{l v} \sin \theta a(a \cos \alpha-a \cos \varphi) d \alpha \\
& =2 a^{2} \gamma_{l v} \sin \theta(\sin \varphi-\varphi \cos \varphi) \\
& =2 a \gamma_{l v} \sin \theta\left(\sqrt{a^{2}-x^{2}}-x \arccos \frac{x}{a}\right),
\end{aligned}
$$

where $\varphi=\arccos (x / a)$, and

$$
\begin{aligned}
M_{2}= & -2 \Delta p \int_{a \cos \varphi}^{a} \int_{0}^{\sqrt{a^{2}-\xi^{2}}}(\xi-a \cos \varphi) d \xi d \eta \\
= & -\frac{4 \gamma_{l v} \sin \theta}{a}\left[-\frac{\pi}{4} a^{2} x+\frac{1}{3}\left(a^{2}-x^{2}\right)^{3 / 2}\right. \\
& \left.+\frac{1}{2} x^{2} \sqrt{a^{2}-x^{2}}+\frac{1}{2} a^{2} x\left(\frac{\pi}{2}-\arccos \frac{x}{a}\right)\right],
\end{aligned}
$$

where $\Delta p=2 \gamma_{l v} / r=2 \gamma_{l v} \sin \theta / a$ is the Laplace pressure. The resultant torque of Eq. (24) is 
$M=M_{1}+M_{2}=\frac{2 \gamma_{l v} \sin \theta}{3 a}\left(a^{2}-x^{2}\right)^{3 / 2}$.

Then the resultant torque on the MCL is given as

$M= \begin{cases}0, & x \leqslant-a, \\ \frac{2 \gamma_{l v} \sin \theta}{3 a}\left(a^{2}-x^{2}\right)^{3 / 2}, & -a \leqslant x \leqslant a, \\ 0, & x \geqslant a .\end{cases}$

Using the basic MCL deflection equation, we have

$\frac{d^{2} z}{d x^{2}}=\frac{M}{E I}=\frac{2 \gamma_{l v} \sin \theta}{3 E I a}\left(a^{2}-x^{2}\right)^{3 / 2}, \quad-a \leqslant x \leqslant a$.

Integrating Eq. (27) once, we obtain

$$
\begin{aligned}
\frac{d z}{d x}= & \frac{2 \gamma_{l v} \sin \theta}{3 E I a}\left[\frac{x}{4}\left(a^{2}-x^{2}\right)^{3 / 2}+\frac{3}{8} a^{2} x \sqrt{a^{2}-x^{2}}\right. \\
& \left.+\frac{3 a^{4}}{8} \arctan \left(\frac{x}{\sqrt{a^{2}-x^{2}}}\right)+\frac{3}{16} \pi a^{4}\right],
\end{aligned}
$$

then the inclination at $x \geqslant a$ is

$\frac{d z}{d x}=\frac{\pi a^{3} \gamma_{l v} \sin \theta}{4 E I}=\frac{3 \pi a^{3} \gamma_{l v} \sin \theta}{E w d^{3}}$.

Integrating Eq. (29) once and we can get the deflection of the MCL given by:

$$
\begin{aligned}
z= & \frac{\gamma_{l v} \sin \theta}{12 E I a}\left[3 a^{4} \sqrt{a^{2}-x^{2}}-a^{2}\left(a^{2}-x^{2}\right)^{3 / 2}\right. \\
& \left.-\frac{2}{5}\left(a^{2}-x^{2}\right)^{5 / 2}+3 a^{4} x\left(\frac{\pi}{2}+\arctan \frac{x}{\sqrt{a^{2}-x^{2}}}\right)\right] .
\end{aligned}
$$

Then the deflection at $x=a$ is

$z(x=a)=\frac{\pi a^{4} \gamma_{l v} \sin \theta}{4 E I}=\frac{3 \pi a^{4} \gamma_{l v} \sin \theta}{E w d^{3}}$,

and the deflection at the segment $a \leqslant x \leqslant L-l$ is

$z(x)=z(x=a)+(x-a) \frac{d z}{d x}(x=a)=\frac{3 \pi a^{3} x \gamma_{l v} \sin \theta}{E w d^{3}}$.

Therefore, the deflection at the free end is

$z(L-l)=\frac{3 \pi a^{3} \gamma_{l v} \sin \theta}{E w d^{3}}(L-l)$.

Now we use FEM to simulate this question. The parameters are listed as below:

$E=1 \mathrm{MPa}, \quad \gamma_{l v}=0.072 \mathrm{~N} / \mathrm{m}, \quad \theta=90^{\circ}$,

$L=1000 \mu \mathrm{m}, \quad l=650 \mu \mathrm{m}, \quad a=40 \mu \mathrm{m}$,

$w=100 \mu \mathrm{m}, \quad d=40 \mu \mathrm{m}$.

From above theoretical analysis, we know the inclination at $x \geqslant$ $a$ and the free-end deflection, respectively, are

$\frac{d z}{d x}(x \geqslant a) \approx 0.00679$,

and

$z(x=L-l)=\frac{3 \pi a^{3} \gamma_{l v} \sin \theta}{E w d^{3}}(L-l) \approx 2.375 \mu \mathrm{m}$.

Fig. 12 presents the numerical results of the vertical displacement of the midline at the upper surface of the MCL induced by a spherical droplet. In addition, the nephogram of the deflection is illustrated in Fig. 13. There is a very small ridge at the rim of the droplet because the vertical component of the liquid-vapor interface tension acts there. And the numerical results of the inclination and the deflection at the free end are approximately 0.00674 and $2.350 \mu \mathrm{m}$, respectively. Therefore, the simulation agrees well with the theoretic solutions.

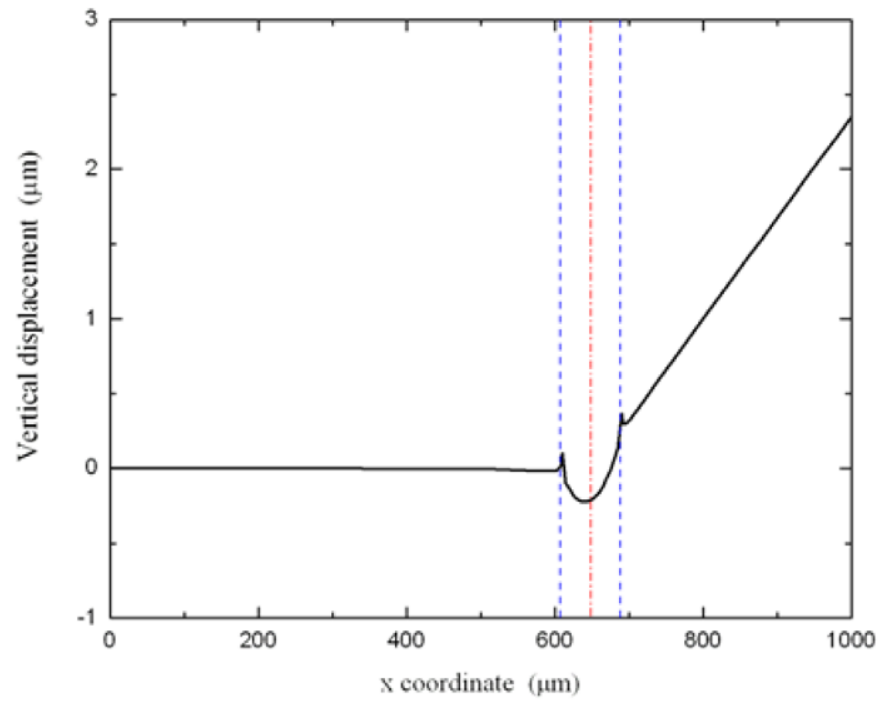

Fig. 12. Vertical displacement of a MCL induced by a spherical droplet.

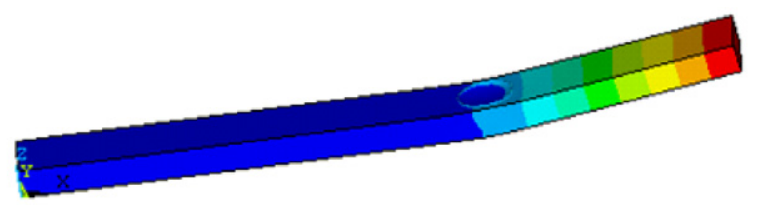

Fig. 13. Nephogram of vertical displacement distribution.

\subsection{Spherical droplet sitting at arbitrary position on MCL}

Now we consider a more general circumstance that the center of the droplet is not located at the longitudinal midline of the MCL illustrated in Fig. 14, in which $x$-axis is the midline and the center is located at $y$-axis. Suppose the distance from the contact center to the midline is $e$, then in addition to the torque which makes the cantilever bending, there is another torque, which will make the MCL twist, the four components of the torque are:

$$
\begin{aligned}
M_{x 1}= & -\int_{-\phi}^{-\alpha} \gamma_{l v} \sin \theta|e+a \sin \varphi| a d \varphi \\
= & -\gamma a^{2} \sin \theta[(\alpha-\phi) \sin \alpha+\cos \alpha-\cos \phi] \\
= & -\gamma_{l v} a \sin \theta\left[e\left(\arcsin \frac{e}{a}-\arccos \frac{x}{a}\right)+\sqrt{a^{2}-e^{2}}-x\right], \\
M_{x 2}= & \frac{2 \gamma_{l v} \sin \theta}{a} \int_{x}^{\sqrt{a^{2}-e^{2}}} 0 . \sqrt{a^{2}-x^{2}}|\mathrm{y}| d y d x \\
= & \frac{\gamma_{l v} \sin \theta}{a}\left[\left(e^{2}+a^{2}\right)\left(\sqrt{a^{2}-e^{2}}-x\right)\right. \\
& -\frac{1}{3}\left(a^{2}-e^{2}\right)^{3 / 2}+\frac{1}{3} x^{3}-e^{2} \sqrt{a^{2}-e^{2}} \\
& \left.+e x \sqrt{a^{2}-x^{2}}-e a^{2}\left(\arctan \frac{\sqrt{a^{2}-e^{2}}}{e}-\arcsin \frac{x}{a}\right)\right],
\end{aligned}
$$




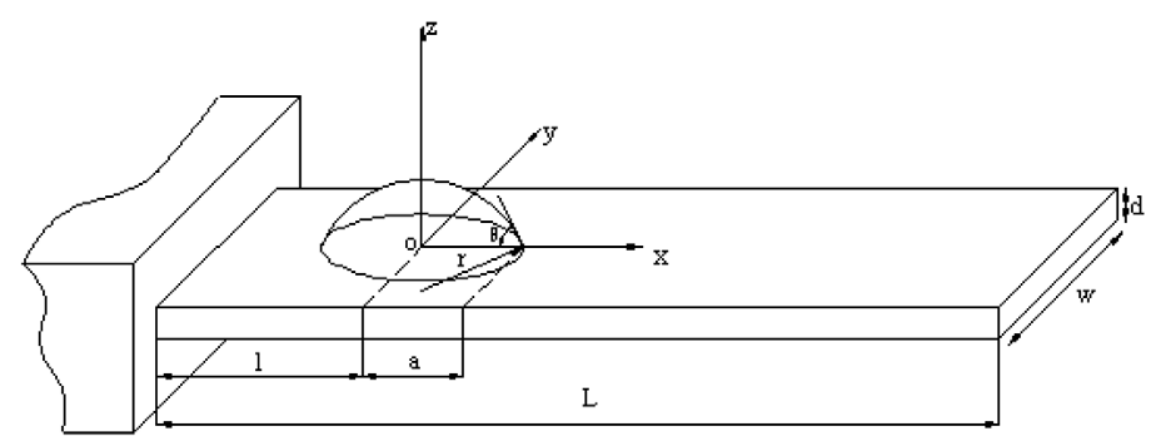

(a)

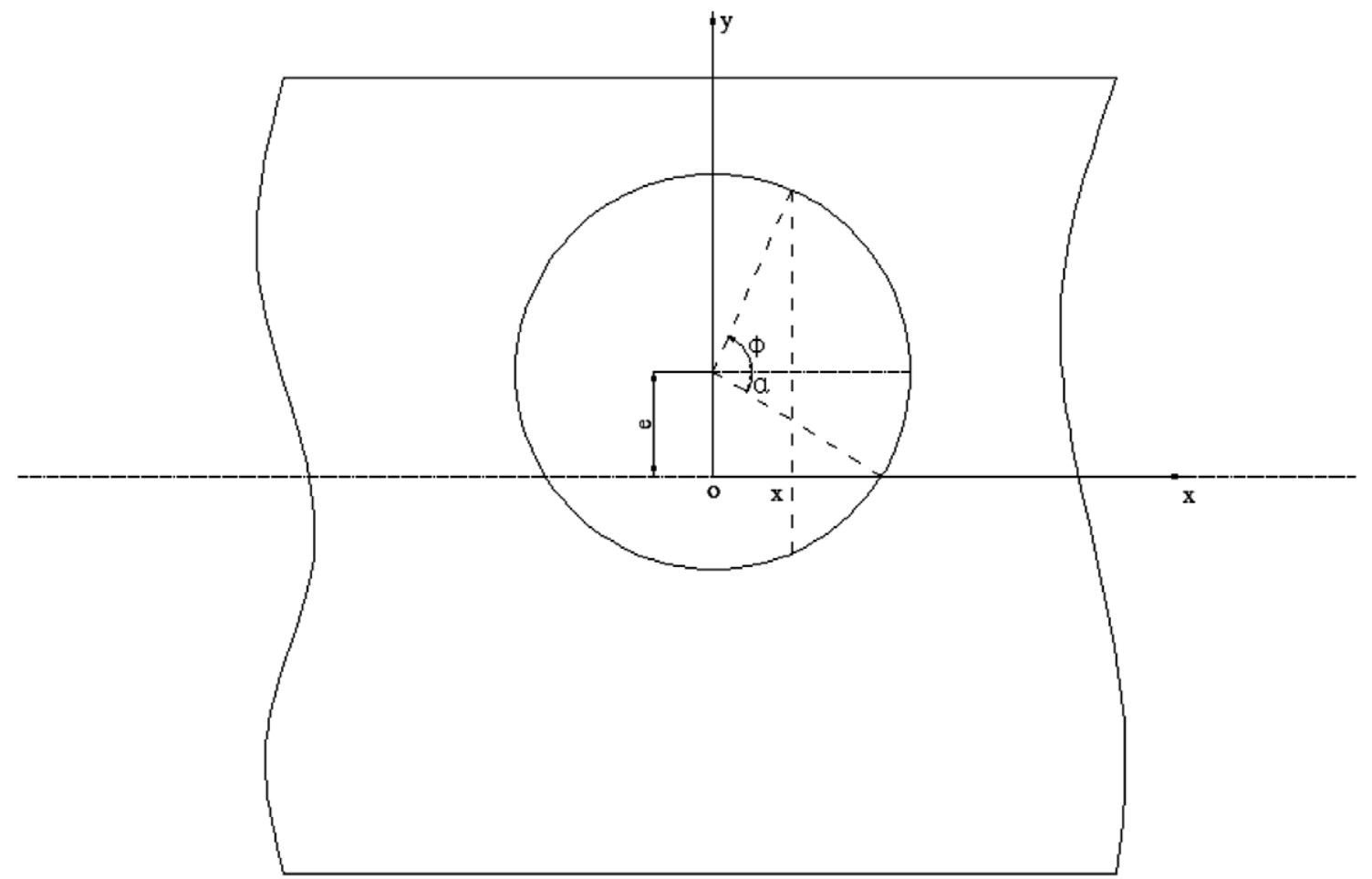

(b)

Fig. 14. Spherical water droplet placed on a MCL.

$$
\begin{aligned}
& M_{x 3}=\int_{-\alpha}^{\phi} \gamma_{l v} \sin \theta(a \sin \varphi+e) a d \varphi \\
& =\gamma_{l v} a \sin \theta\left(\sqrt{a^{2}-e^{2}}-x+e \arccos \frac{x}{a}+e \arcsin \frac{e}{a}\right), \\
& M_{x 4}=-\frac{2 \gamma_{l v} \sin \theta}{a} \int_{x}^{\sqrt{a^{2}-e^{2}}} e+\sqrt{a^{2}-x^{2}} \int_{0} y d y d x \\
& -\frac{2 \gamma \sin \varphi}{a} \int_{\sqrt{a^{2}-e^{2}}}^{a} \int_{e-\sqrt{a^{2}-x^{2}}}^{e+\sqrt{a^{2}-x^{2}}} y d y d x \\
& =-\frac{\gamma_{l v} \sin \theta}{a}\left[e a^{2}\left(\pi-\arctan \frac{\sqrt{a^{2}-e^{2}}}{e}\right)\right. \\
& -e^{2} x-a^{2} x+a^{2} \sqrt{a^{2}-e^{2}}-e\left(x \sqrt{a^{2}-x^{2}}+a^{2} \arcsin \frac{x}{a}\right) \\
& \left.+\frac{1}{3} x^{3}-\frac{1}{3}\left(a^{2}-e^{2}\right)^{3 / 2}\right]
\end{aligned}
$$

$$
\text { where } \alpha=\arccos (e / a) \text { and } \varphi=\arccos (x / a) \text {. }
$$

The resultant twist torque is

$$
M_{x}=M_{x 1}+M_{x 2}+M_{x 3}+M_{x 4}=\frac{2 e \gamma_{l v} \sin \theta}{a} x \sqrt{a^{2}-x^{2}},
$$

and the distribution of the twist torque on the MCL is

$M_{x}= \begin{cases}0, & x \leqslant-a, \\ \frac{2 e \gamma_{l v} \sin \theta}{a} x \sqrt{a^{2}-x^{2}}, & -a \leqslant x \leqslant a, \\ 0, & x \geqslant a .\end{cases}$

The twist angle in the case of a rectangular cross section is [39]

$\frac{d \phi}{d x}=\frac{M_{x}}{\beta w d^{3} G}$,

then by integrating Eq. (37), we express the twist angle in a quite straightforward manner as

$\phi= \begin{cases}0, & x \leqslant-a, \\ -\frac{2 e \gamma_{l v} \sin \theta}{3 a \beta w d^{3} G}\left(a^{2}-x^{2}\right)^{3 / 2}, & -a \leqslant x \leqslant a, \\ 0, & x \geqslant a .\end{cases}$

The values of twist of the numerical factor $\beta$ are given in Table 1 [39]. The deflection of a point $(x, y, 0)$ can be written as 
Table 1

Data for the twist of a shaft of rectangular cross section

\begin{tabular}{llllllll}
\hline$w / d$ & 1.00 & 1.50 & 2.00 & 3.00 & 4.00 & 6 & 10 \\
$\beta$ & 0.141 & 0.196 & 0.229 & 0.263 & 0.281 & 0.299 & 0.313 \\
\hline
\end{tabular}

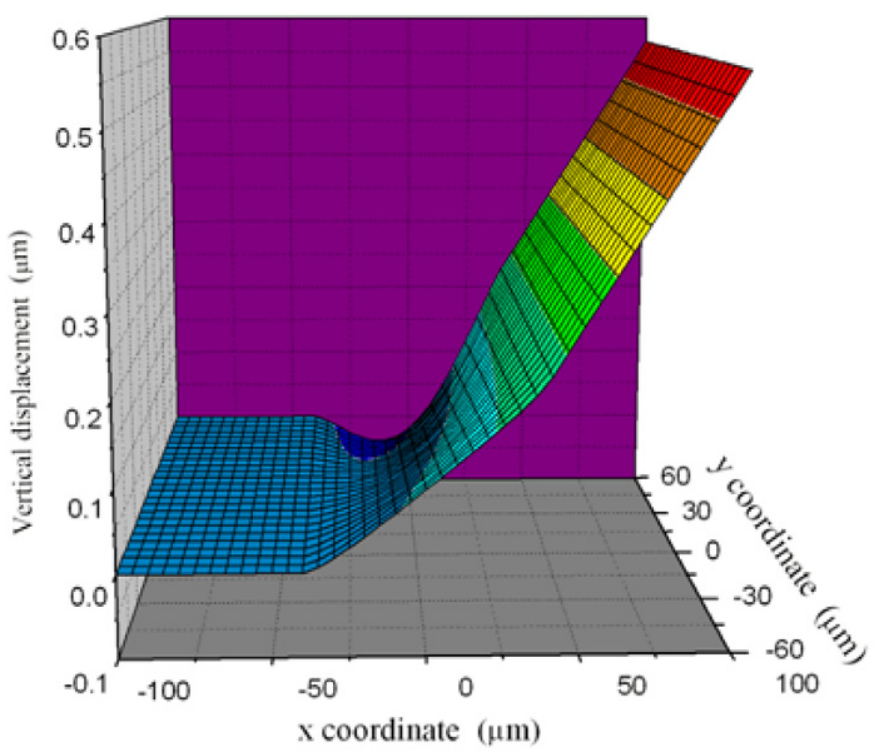

Fig. 15. Vertical displacement of MCL induced by a spherical droplet whose center is not sitting at the midline of its upper surface.

$z(x, y, 0)=z_{b}+z_{t}$,

where $z_{b}$ denotes the deflection induced by bending given in Section 4.2 and

$z_{t}=y \sin \phi$

The parameters for the numerical simulation are listed below:

$$
\begin{aligned}
& E=1 \mathrm{MPa}, \quad v=0.3, \quad \gamma_{l v}=0.072 \mathrm{~N} / \mathrm{m}, \quad \theta=90^{\circ}, \\
& L=1000 \mu \mathrm{m}, \quad l=650 \mu \mathrm{m}, \quad a=40 \mu \mathrm{m}, \\
& w=120 \mu \mathrm{m}, \quad d=40 \mu \mathrm{m}, \quad e=10 \mu \mathrm{m} .
\end{aligned}
$$

From Eqs. (38) and (40), we know the maximum of twist angle and corresponding deflection are, respectively,

$\phi_{\text {min }} \approx-0.001$ and $\max \left(z_{t}\right)=z_{t}(0,-60,0) \approx 0.06 \mu \mathrm{m}$.

Since $y$ coordinates of points at the midline are zero, the additional deformations of these points induced by twisting are all zero theoretically, that is, the total deflection of the midline at the upper surface induced by both bending and twisting is the same as that in Section 4.2. In addition, 3-D numerical results of the cantilever deflection are given partly as shown in Fig. 15. From this figure, we can find that the additional deformation of the MCL induced by twisting is so small that its effect can be neglected. The nephogram of the MCL displacement in the normal direction is given in Fig. 16. From this figure, we can find that the maximum vertical displacement is approximately $1.96 \mu \mathrm{m}$.

If there are several microdrops, which are either cylindrical or spherical, we can easily use the superposition method to calculate the inclination and deflection of MCL.

\section{Summary}

The effects of a liquid droplet on the deformation of PDMS membrane and a MCL were investigated. The deformation of PDMS membrane, induced by surface tension and Laplace pressure, has

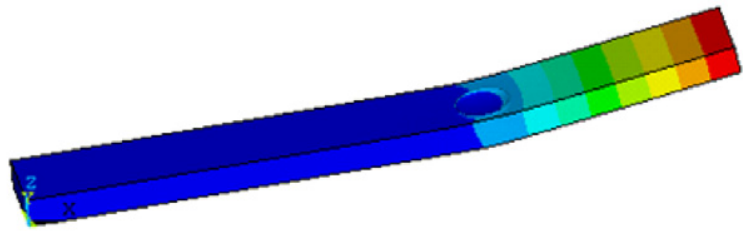

Fig. 16. Nephogram of vertical displacement distribution.

been calculated using FEM with both MR model and linear elastic model. Since the deformation is very small, the solutions with linear elastic model are nearly the same to that with MR model. That is to say, although PDMS is a hyperelastic material, a linear elastic model is still valid when considering the role of a droplet on the deformation of PDMS membrane.

Theoretical study on the deformation of a MCL induced by a liquid droplet shows that: When there is one or several liquid droplets sitting on a MCL, the cantilever will deflect due to the vertical component of liquid-vapor interface tension and Laplace pressure, and it is proportional to $\gamma_{l v} \sin \theta /\left(E d^{3}\right)$. When the center of a droplet is not located at the midline of the upper surface, there will be a partial twist; however, it has little effect on the deflection at the free end. When the liquid droplet is nearer to the fixed end, the deflection on the free end will be larger and the maximum of the deflection cannot be neglected.

\section{Acknowledgments}

This work was jointly supported by the National Basic Research Program of China (973 Program, Grant No. 2007CB310500) and the National Natural Science Foundation of China (NSFC, Grant Nos. 10772180 and 10721202). Drs. Zhi-Qiao Wang, Zhenyu Yang and Zai-Bin Cheng are gratefully acknowledged for their helpful discussions.

\section{References}

[1] C.H. Wang, G.B. Lee, Biosens. Bioelectron. 21 (2005) 419-425.

[2] A. Mata, A.J. Fleischman, S. Roy, Biomed. Microdevices 7 (2005) 281-293.

[3] H. Takao, K. Miyamura, H. Ebi, M. Ashiki, K. Sawada, M. Ishida, Sens. Actuators A 119 (2005) 468-475.

[4] N.S. Tambe, B. Bhushan, Ultramicroscopy 105 (2005) 238-247.

[5] I. Byun, J. Yang, S. Park, Microelectron. J. 39 (2008) 717-722.

[6] T. Suzuki, Y. Teramura, H. Hata, K. Inokuma, I. Kanno, H. Iwata, H. Kotera, Microsyst. Technol. 13 (2007) 1391-1396.

[7] J.H. Kim, K.H. Na, C.J. Kang, Y.S. Kima, Sens. Actuators A 120 (2005) 365-369.

[8] O.C. Jeong, S.W. Park, S.S. Yang, J.J. Park, Sens. Actuators A 123/124 (2005) 453458.

[9] T. Pan, S.J. McDonald, E.M. Kail, B. Ziaiel, J. Micromech. Microeng. 15 (2005) 286-294.

[10] D.C. Duffy, O.J.A. Schueller, S.T. Brittain, G.M. Whitesides, J. Micromech. Microeng. 9 (1999) 211-217.

[11] K. Kosokawa, R. Maeda, J. Micromech. Microeng. 10 (2000) 415-420.

[12] H.P. Chou, M.A. Unger, S.R. Quake, Biomed. Microdevices 3 (2001) 323-330.

[13] K. Lim, S. Kim, J.H. Hahn, Sens. Actuators B 92 (2003) 208-214.

[14] J.C. Lötters, W. Olthuis, P.H. Veltink, P. Bergveld, J. Micromech. Microeng. 7 (1997) 145-147.

[15] D. Armani, C. Liu, N. Aluru, in: Proceedings of 12th International Conference on MEMS (MEMS' 99), Orlando, FL, USA, pp. 222-227.

[16] R.C. Huang, L. Anand, unpublished work.

[17] F. Schneider, T. Fellner, J. Wilde, U. Wallrabe, J. Micromech. Microeng. 18 (2008) 065008.

[18] M. Mooney, J. Appl. Phys. 11 (1940) 582-592.

[19] R.S. Rivlin, D.W. Saunders, Philos. Trans. R. Soc. A 243 (1951) 251-288.

[20] R.W. Ogden, Non-Linear Elastic Deformations, Dover, New York, 1997. 
[21] L.R.G. Treloar, The Physics of Rubber Elasticity, Oxford University Press, Oxford, 1958.

[22] W.W. Feng, P. Huang, J. Appl. Mech. Trans. ASME 41 (1974) 767-771.

[23] D.C. Pamplona, P.B. Gonçalves, S.R.X. Lopes, Int. J. Solids Struct. 48 (2006) 683696.

[24] G.J. Nam, K.H. Ahn, J.W. Lee, Polym. Eng. Sci. 40 (2000) 2232-2240.

[25] K. Bellamy, G. Limbert, M.G. Waters, J. Middleton, Biomaterials 24 (2003) 50615066.

[26] Y. Dong, R.J.T. Lin, D. Bhattacharyya, J. Mater. Sci. 40 (2005) 399-410.

[27] Y.S. Yu, Z.Y. Yang, Y.P. Zhao, J. Adhes. Sci. Technol. 22 (2008) 687-698.

[28] T. Kawamura, K. Urayama, S. Kohjiya, J. Soc. Rheol. 31 (2003) 213-217.

[29] W. Oppermann, N. Rennar, Prog. Colloid Polym. Sci. 75 (1987) 49-54.

[30] R.H. Finney, A. Kumar, Rubber Chem. Technol. 61 (1988) 879-891.

[31] A.N. Gent, Engineering with Rubber - How to Design Rubber Components, Hanser Gardner Publications, 2000.
[32] H. Jensenius, J. Thaysen, A.A. Rasmussen, L.H. Veje, O. Hansen, A. Boisen, Appl. Phys. Lett. 76 (2000) 2615-2617.

[33] E. Bonaccurso, H.J. Butt, J. Phys. Chem. B 109 (2005) 253-263.

[34] T. Haschke, E. Bonaccurso, H.J. Butt, D. Lautenschlager, F. Schönfeld, W. Wiechert, J. Micromech. Microeng. 16 (2006) 2273-2280.

[35] S. Jeon, R. Desikan, F. Tian, T. Thundat, Appl. Phys. Lett. 88 (2006) 103118.

[36] H.J. Lee, Y.S. Chang, Y.P. Lee, K.H. Jeong, H.Y. Kim, Sens. Actuators A 136 (2007) 717-722.

[37] D.S. Golovko, P. Bonanno, S. Lorenzoni, F. Stefani, R. Raiteri, E. Bonaccurso, J. Micromech. Microeng. 18 (2008) 095029.

[38] X.P. Zheng, H.P. Zhao, L.T. Gao, J.L. Liu, S.W. Yu, X.Q. Feng, J. Colloid Interface Sci. 323 (2008) 133-140.

[39] S. Timoshenko, Strength of Materials: Part I, Elementary Theory and Problems, 3rd ed., Van Nostrand, New York, 1955, p. 290. 